\title{
Synergistic induction of apoptosis by sulindac and simvastatin in A549 human lung cancer cells via reactive oxygen species-dependent mitochondrial dysfunction
}

\author{
KI-EUN HWANG $^{1 *}$, CHUL PARK $^{1 *}$, SU-JIN KWON ${ }^{1}$, YOUNG-SUK KIM $^{1}$, DO-SIM PARK ${ }^{2}$, MI-KYUNG LEE ${ }^{3}$, \\ BYOUNG-RYUN KIM ${ }^{4}$, SEONG-HOON PARK ${ }^{5}$, KWON-HA YOON ${ }^{5}$, EUN-TAIK JEONG ${ }^{1}$ and HAK-RYUL KIM ${ }^{1}$ \\ ${ }^{1}$ Department of Internal Medicine, ${ }^{2}$ Laboratory Medicine, Institute of Wonkwang Medical Science, \\ Departments of ${ }^{3}$ Thoracic and Cardiovascular Surgery, ${ }^{4}$ Obstetrics and Gynecology and ${ }^{5}$ Radiology, \\ Wonkwang University, School of Medicine, Iksan, Jeonbuk 570-749, Republic of Korea
}

Received February 22, 2013; Accepted April 15, 2013

DOI: 10.3892/ijo.2013.1933

\begin{abstract}
Prevention of lung cancer is more feasible and holds greater promise when different agents are used in combination to target multiple processes during carcinogenesis. The mechanisms by which non-steroidal anti-inflammatory drugs and statins inhibit cancer cell growth and induce apoptosis are not fully understood. This study was designed to investigate lung cancer chemoprevention through a mechanism-based approach using sulindac at low doses in combination with simvastatin. We found that sulindac-induced cytotoxicity was significantly enhanced in the presence of simvastatin. The combination of sulindac and simvastatin induced more extensive caspasedependent apoptosis in A549 cells compared to that induced with either drug alone. The combination of sulindac and simvastatin also increased the loss of mitochondrial transmembrane potential $\left(\Delta \Psi_{\mathrm{m}}\right)$ and the cytosolic release of cytochrome $c$. In addition, ROS generation in cells treated with both sulindac and simvastatin was markedly increased compared to cells treated with either sulindac or simvastatin alone. The enhancement of ROS generation by sulindac and simvastatin was abrogated by pretreatment with NAC, which also prevented apoptosis and mitochondrial dysfunction induced by sulindac and simvastatin. These results suggest that sulindac and simvastatin-induced ROS generation in A549 lung cancer cells causes their accumulation in mitochondria, triggering the release of apoptogenic molecules from the mitochondria to the cytosol, and thus leading to caspase activation and cell death.
\end{abstract}

Correspondence to: Dr Hak-Ryul Kim, Department of Internal Medicine, Institute of Wonkwang Medical Science, Wonkwang University, School of Medicine, 344-2 Shinyong-dong, Iksan, Jeonbuk 570-749, Republic of Korea

E-mail: kshryj@wku.ac.kr

${ }^{*}$ Contributed equally

Key words: sulindac, simvastatin, A549 cells, apoptosis, reactive oxygen species

\section{Introduction}

Many new therapeutic maneuvers during the last decade relates to the introduction of novel biologically targeted therapeutic agents, improving surgical techniques, and increased utilization of concomitant chemoradiotherapy for locally advanced lung cancer (1). Despite this, the success rates for treatment remain quite dismal, and the limited options for the management of lung cancer have necessitated the search for novel preventive approaches for this disease. The advantages of using a combinatorial chemopreventive approach, in which 2 or more critical molecules are simultaneously targeted, include lower dose requirements, reduced incidence of associated toxicities, and increased likelihood of human acceptability (2-5).

Non-steroidal anti-inflammatory drugs (NSAIDs) have attracted substantial attention after the discovery that sulindac induces the regression of colon adenomatous polyps in cancer therapy (6). Sulindac, a structural isoform of indomethacin, exerts antiproliferative and apoptotic effects, which eventually lead to the regression of cancer cells $(7,8)$. Many studies have revealed a link between COX-2 expression and carcinogenesis, suggesting that COX-2 inhibition can prevent cancer growth or progression (9). However, sulindac sulfone, a metabolite of sulindac that lacks the ability to inhibit COX-2, reduces the incidence of tumors in breast and colon cancer $(10,11)$, indicating the involvement of other mechanisms such as the production of reactive oxygen species (ROS) and the inhibition of NFKB-mediated signals $(12,13)$.

Statins are a class of drugs that inhibit the rate-limiting step of the mevalonate pathway, which is catalyzed by 3-hydroxy-3-methylglutaryl coenzyme A (HMG-CoA) reductase (14). Besides their lipid-lowering effect, statins have been studied for their anticarcinogenic properties in various cancer cell types, including carcinomas of the colon, prostate, breast, lung and skin $(15,16)$, which may make them relevant to cancer prevention or treatment. Many studies have shown that the antiproliferative and proapoptotic effects of statins are more pronounced in malignant than in non-malignant cells $(17,18)$. Moreover, low concentrations of statins have 
been shown to sensitize cancer cell lines to cytostatic drugs such as 5-fluorouracil (5-FU), taxol, etoposide, doxorubicin and cisplatin (19-22).

The relatively high dose required for the observed chemopreventive effect of NSAIDs in lung cancer patients may discourage their individual use on a long-term basis for lung cancer prevention due to the potentially increased risk of serious gastrointestinal and cardiovascular side-effects (23-25). However, lower doses of NSAIDs may prove to be more beneficial in the prevention of lung cancer when administered in combination with other complementary agents. For example, many reports have indicated that NSAIDs possess synergistic effects in combination with other therapeutics such as statins, PPAR $\gamma$ ligands, epidermal growth factor receptor tyrosine kinase inhibitors (EGFR-TKI) and TRAIL receptor ligands (26).

Recently, considerable evidence has suggested that combination treatment of NSAIDs and statin synergistically induced apoptosis in cancer cells $(27,28)$. However, the mechanism underlying deregulated survivin by NSAIDs and statin on human non-small cell lung cancer cells has not been elucidated. Our results suggest that a mechanism-based approach, using sulindac at low doses in combination with simvastatin, has potential in the chemoprevention of lung cancer.

\section{Materials and methods}

Materials. RPMI-1640, fetal bovine serum (FBS), and antibiotics were obtained from Gibco-BRL Co (Grand Island, NY). Sulindac, simvastatin, 3-(4,5-dimethyl-2-thiazolyl)-2,5-diphenyl-2H-tetrazolium bromide (MTT), propidium iodide (PI), bicinchoninic acid, dimethyl sulfoxide (DMSO) and $\mathrm{N}$-acetylcysteine (NAC) were purchased from Sigma-Aldrich (St. Louis, MO). JC-1 was obtained from Molecular Probes (Eugene, OR). Primary antibodies against the following targets were used: caspase-3, -8 and -9 ; poly(ADP-ribose) polymerase (PARP); Puma; Bim; Mcl-1; Bcl-XL; and XIAP (Santa Cruz Biotechnology, Santa Cruz, CA); serine/threonine protein kinase (Akt); phospho-Akt; JNK; phosphor-JNK; p38; phospho-p38; survivin; GAPDH (Cell Signaling Technology, Beverly, MA); and cytochrome $c$ (Pharmingen, San Diego, CA). Anti-rabbit IgG-conjugated horseradish peroxidase (HRP) antibodies and enhanced chemiluminescent (ECL) kit were purchased from Amersham Pharmacia Biotech (Buckinghamshire, UK).

Cell culture and viability test. A549 human lung cancer cells were obtained from the Korean Cell Line Bank (Seoul, Korea) and grown in RPMI-1640 containing $100 \mathrm{U} / \mathrm{ml}$ penicillin, $0.1 \mathrm{mg} / \mathrm{ml}$ streptomycin and $10 \% \mathrm{FBS}$, and they were maintained in a humidified atmosphere of $5 \% \mathrm{CO}_{2}$ in air at $37^{\circ} \mathrm{C}$ and maintained in the $\log$ phase. Cell viability was determined by measuring the mitochondrial conversion of MTT to a colored product. Cells were treated with the specified drugs. To determine cell viability, MTT was added to the cell suspension for $4 \mathrm{~h}$. After 3 washes with phosphatebuffered saline (PBS; pH 7.4), the insoluble formazan product was dissolved in DMSO. The optical density (OD) of each well was then measured using a microplate reader (Titertek Multiskan; Flow Laboratories, North Ryde, Australia) at
$590 \mathrm{~nm}$. The OD resulting from formazan production in control cells was considered as $100 \%$ cell viability, and all other measurements were expressed as a percentage of the control cell value.

Annexin V assay for the assessment of apoptosis. A549 cells undergoing early/late apoptosis were analyzed by Annexin V-FITC and PI staining. In all, $2.5 \times 10^{5}$ cells in the exponential growth phase were seeded in $60-\mathrm{mm}^{2}$ dishes. Cells were left untreated or incubated with specified drugs for the indicated times at $37^{\circ} \mathrm{C}$. Both adherent and floating cells were collected and analyzed by the Annexin V assay, according to the manufacturer's instructions. Pelleted cells were briefly washed with PBS and resuspended in an Annexin binding buffer (BD Pharmingen). Cells were then incubated with Annexin V-phycoerythrin and propidium iodide for $15 \mathrm{~min}$ at room temperature. After incubation, the stained cells were analyzed using a FACScan system equipped with Cell Quest software (Becton-Dickinson, San Jose, CA). Cells with no drug treatment were used as controls.

Measurement of the mitochondrial membrane potential $\left(\Delta \Psi_{m}\right)$. Cells were harvested at the indicated treatment time points, washed with PBS, and then stained with $10 \mu \mathrm{g} / \mathrm{ml} \mathrm{JC}-1$ at $37^{\circ} \mathrm{C}$ for $30 \mathrm{~min}$. After a brief wash with serum-free medium, cells were immediately analyzed using a FACScan system equipped with a Cell Quest software (Becton-Dickinson). At low concentrations, JC-1 exists mainly in a monomeric form, emitting green fluorescence (emission maximum at $530 \mathrm{nM}$ ), whereas at higher concentrations it forms aggregates, known as J-aggregates, which emit orange-red fluorescence (emission maximum at $590 \mathrm{nM}$ ).

Measurement of reactive oxygen species. A549 cells were incubated in the dark with $10 \mu \mathrm{mol} / 1$ 5- (and -6)-carboxy-2',7'dichlorodihydrofluorescein diacetate, carboxy- $\mathrm{H}_{2}$ DCFDA (Molecular Probes) for $30 \mathrm{~min}$. Cells were then washed, scraped gently, resuspended in PBS, and kept on ice for immediate detection by FACScan flow cytometry using an argon laser $(488 \mathrm{~nm})$ for excitation. Green fluorescence due to intracellularly trapped DCF was collected on the FL1 channel on a log scale. Data were acquired and analyzed using the Cell Quest program (Becton-Dickinson).

Western blot analysis. Cells were harvested and lysed using radioimmunoprecipitation assay buffer [50 mM Tris- $\mathrm{Cl}$ (pH 7.4), 1\% NP-40, $150 \mathrm{mM} \mathrm{NaCl}, 1 \mathrm{mM}$ EDTA, $1 \mathrm{mM}$ phenylmethylsulfonyl fluoride, $1 \mu \mathrm{g} / \mathrm{ml}$ each of aprotinin and leupeptin, and $\left.1 \mathrm{mM} \mathrm{Na} \mathrm{VO}_{4}\right]$. After centrifugation at $12,000 \mathrm{x} \mathrm{g}$ for $30 \mathrm{~min}$, the supernatant was collected, and the protein concentration was determined by the Lowry method (29). Equal amounts of protein were separated on $12 \%$ sodium dodecyl sulfate-polyacrylamide gel electrophoresis (SDS-PAGE) gels under reducing conditions and subsequently transferred to nitrocellulose membranes. Membranes were blocked with 5\% skim milk in TBS-T [25 mM Tris (pH 7.6), $138 \mathrm{mM} \mathrm{NaCl}$ and $0.05 \%$ Tween-20] for $1 \mathrm{~h}$ and probed with primary antibodies (at 1:1,000-1:5,000). After a series of washes, membranes were further incubated with secondary antibody (at 1:2,000-1:10,000) conjugated with HRP. Detection 
of the immunoreactive signals was carried out using an ECL detection system (Amersham Pharmacia Biotech).

Preparation of cytosolic and mitochondrial fractions. Cytosolic and mitochondrial fractions were prepared as described previously (30) with certain modifications. Briefly, A549 cells were harvested, washed with ice-cold PBS, and then incubated with $500 \mu \mathrm{M}$ buffer A [250 mM sucrose; $20 \mathrm{mM}$ HEPES (pH 7.5); $10 \mathrm{mM} \mathrm{KCl} ; 1.5 \mathrm{mM} \mathrm{MgCl}_{2}$; 1 mM EGTA; 1 mM EDTA; 1 mM DTT; 1 mM PMSF; and $10 \mu \mathrm{g} / \mathrm{ml}$ each of leupeptin, aprotinin and pepstatin A] on ice for $30 \mathrm{~min}$. Cells were then disrupted by 20 passages through a 26 -gauge needle and centrifuged at $750 \mathrm{xg}$ for $10 \mathrm{~min}$. The supernatant was centrifuged at $10,000 \mathrm{x} \mathrm{g}$ for $25 \mathrm{~min}$. After centrifugation, the cytosolic fraction was frozen at $70^{\circ} \mathrm{C}$. The pellet containing mitochondria was washed with ice-cold buffer $\mathrm{A}$ and then resuspended with cell lysis buffer. The resuspended pellet was incubated on ice for $30 \mathrm{~min}$ and then centrifuged at $10,000 \mathrm{x}$ g for $25 \mathrm{~min}$. The supernatant thus collected represented the cytosolic fraction of A549 cells.

Statistical analysis. Each experiment was performed at least 3 times, and all values are represented as the means \pm SD of triplicate samples. The Student's t-test was used to determine the statistical significance of the results. Values of $p<0.05$ were considered statistically significant.

\section{Results}

Effect of sulindac and simvastatin, alone and in combination, on the growth of A549 lung cancer cells. A549 cells were treated with simvastatin after which their viability was measured by the MTT assay. As shown in Fig. 1A, simvastatin is cytotoxic at concentrations equal to or greater than $5 \mu \mathrm{M}$ and up to $48 \mathrm{~h}$ of treatment. We next examined the combined effects of $5 \mu \mathrm{M}$ simvastatin and increasing concentrations of sulindac. In the presence of simvastatin, sulindac-induced cytotoxicity was significantly enhanced. Incubation of A549 cells with $300 \mu \mathrm{M}$ sulindac alone for $48 \mathrm{~h}$ decreased cell viability to $70 \%$, whereas co-treatment with $5 \mu \mathrm{M}$ simvastatin resulted in $48 \%$ viability (Fig. 1B).

Combination of sulindac and simvastatin enhances caspasedependent apoptosis. To examine whether the observed growth inhibition was due to enhanced apoptosis, the proportion of apoptotic cells was determined using Annexin V-propidium iodide staining, which is more sensitive for detecting apoptosis than the methods based on hypodiploid DNA content. Annexin V staining showed that the combination of sulindac and simvastatin significantly enhanced apoptosis compared with individual treatment with either drug. As shown in Fig. 2A, individual treatment with $300 \mu \mathrm{M}$ sulindac or $5 \mu \mathrm{M}$ simvastatin resulted in apoptosis rates of 18.2 and $17.0 \%$, respectively, whereas $48.9 \%$ Annexin V-positive cells were observed after combined treatment with both drugs. To further elucidate the mechanism of apoptosis induced by sulindac and simvastatin, cell lysates were evaluated by immunoblot analysis (Fig. 2B). Our results showed that the combination of sulindac and simvastatin enhanced the expression of the processed $85-\mathrm{kDa}$ isoform of PARP, which is known to play a
$\mathbf{A}$

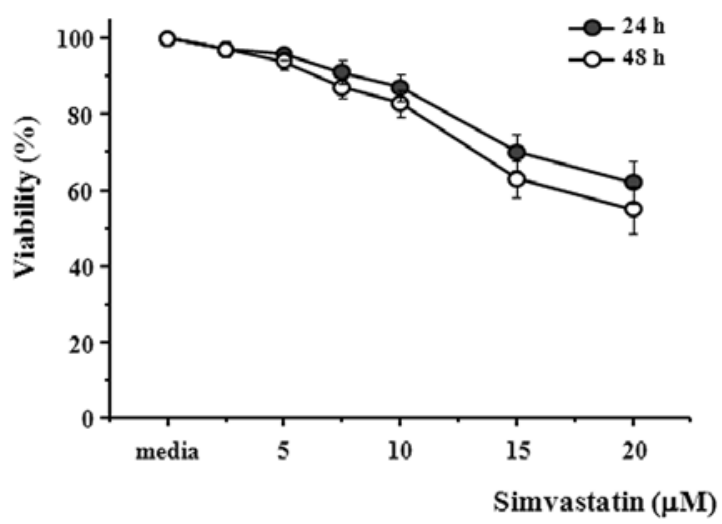

B

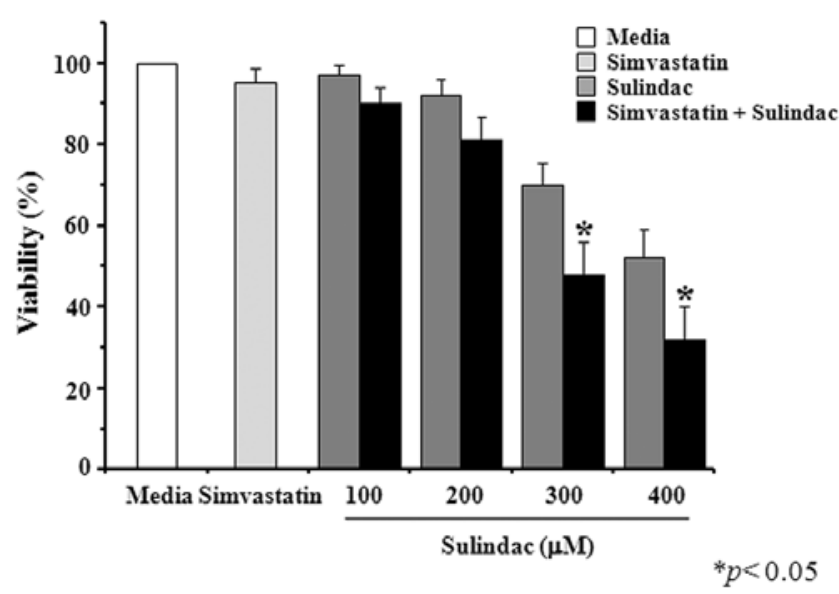

Figure 1. Inhibitory effect of combination treatment with sulindac and simvastatin on cell growth of A549 cells. (A) Cells were treated with 0-20 $\mu \mathrm{M}$ simvastatin for 24 or $48 \mathrm{~h}$, (B) followed by treatment with different concentrations of sulindac in the absence or presence of $5 \mu \mathrm{M}$ simvastatin for $48 \mathrm{~h}$, and viability was then measured by the MTT assay. The viability of control cells was set at $100 \%$, and the cell survival relative to that of the control is presented. The data represent the mean $\pm \mathrm{SD}$ of 3 independent experiments. ${ }^{*} \mathrm{p}<0.05$ compared to the control.

major role in evading the apoptosis process. Moreover, combination of sulindac and simvastatin led to a marked increase in the expression of caspase-3, -8 and -9 . These results indicate that sulindac and simvastatin play a major role in enhancing caspase-dependent apoptosis in A549 cells.

Combination of sulindac and simvastatin leads to mitochondrial dysfunction. To identify components upstream of caspase-3 in apoptotic signaling, the status of markers of mitochondrial dysfunction, including mitochondrial membrane potential transition (MPT) and cytosolic release of cytochrome $c$, was evaluated in cells treated with sulindac and simvastatin. JC-1 is a cationic dye that exhibits potential-dependent accumulation in mitochondria, indicated by a fluorescence emission shift from green $(525 \mathrm{~nm})$ to red $(590 \mathrm{~nm})$. Therefore, JC-1 has been widely used for the detection of apoptosis by mitochondrial depolarization, as indicated by a decrease in the red/green fluorescence intensity ratio (31). As shown in 
A

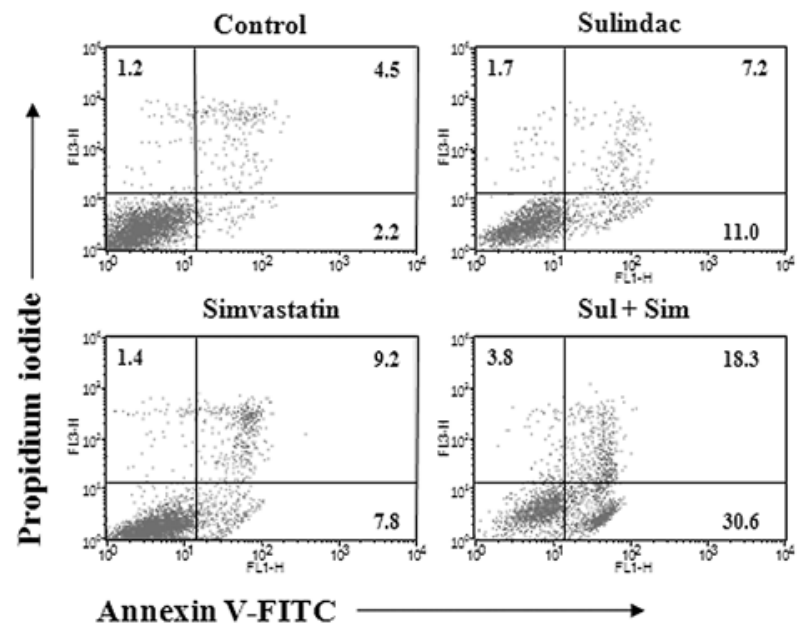

B

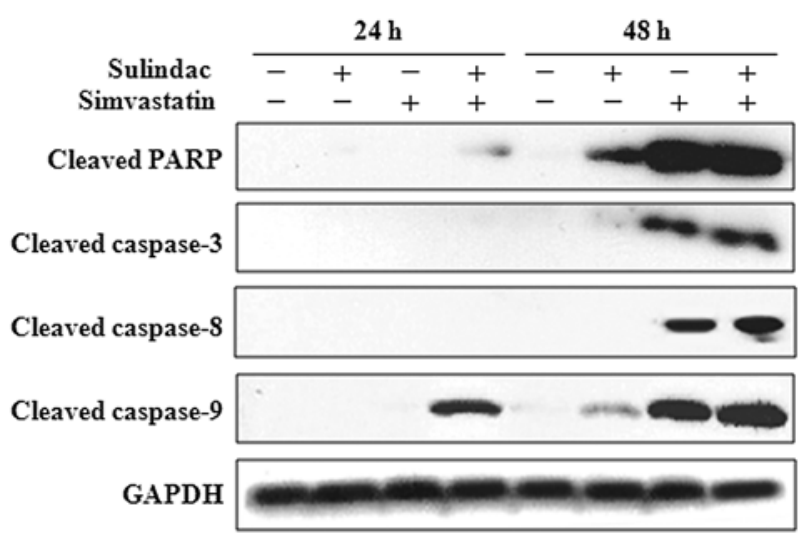

Figure 2. Effect of sulindac and simvastatin on caspase-dependent apoptosis. (A) Cells were incubated with $300 \mu \mathrm{M}$ sulindac and/or $5 \mu \mathrm{M}$ simvastatin for $48 \mathrm{~h}$, and apoptosis was evaluated by green fluorescent protein-Annexin $\mathrm{V}+$ propidium iodide. Percentage of Annexin V and propidium iodide positive cells are indicated for each test condition. (B) Cells were treated with sulindac and simvastatin, alone and in combination, for 24 and $48 \mathrm{~h}$, after which the cel lysate was subjected to $12 \%$ SDS-PAGE to measure the expression of PARP and caspase- $-3,-8$ and -9 . The numerical values from densitometry data were normalized with the loading control and shown below for respective blots.

Fig. 3A, individual treatment with $300 \mu \mathrm{M}$ sulindac or $5 \mu \mathrm{M}$ simvastatin resulted in JC-1 monomer levels of 15.9 and $26.4 \%$, respectively, whereas a JC-1 monomer level of $43 \%$ was observed in cells treated with the drug combination. Since the loss of mitochondrial transmembrane potential $\left(\Delta \Psi_{\mathrm{m}}\right)$ results in cytochrome $c$ release into the cytosol, cytochrome $c$ levels were evaluated by western blot analysis in both mitochondrial and cytosolic fractions (Fig. 3B). Combination treatment with sulindac and simvastatin was associated with an increased cytosolic level of cytochrome $c$ and a corresponding decrease in its mitochondrial level.

Combination of sulindac and simvastatin elicits different effects on survival and stress signaling pathways. To better understand the increased sensitivity of lung cancer cells to a combination treatment with sulindac and simvastatin, we
A

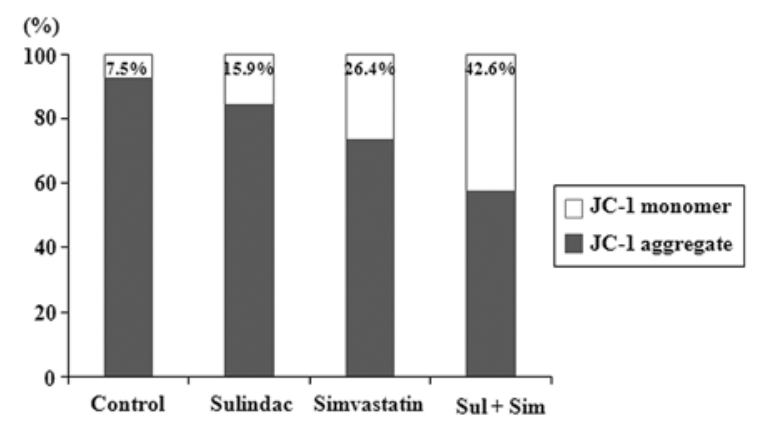

B

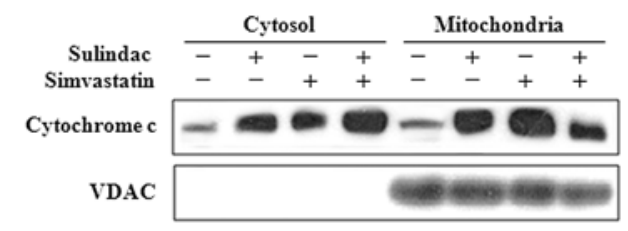

Figure 3. Mitochondrial membrane potential transition (MPT) and cytosolic release of cytochrome $c$ by treatment with sulindac and/or simvastatin. (A) Cells were treated with $300 \mu \mathrm{M}$ sulindac and/or $5 \mu \mathrm{M}$ simvastatin for $36 \mathrm{~h}$, then stained with $10 \mu \mathrm{g} / \mathrm{ml}$ of $\mathrm{JC}-1$, and analyzed by flow cytometry. Percentage of JC-1 monomer are indicated for each test condition. Each panel is representative of 3 identical experiments. (B) Cells were incubated with $300 \mu \mathrm{M}$ sulindac and/or $5 \mu \mathrm{M}$ simvastatin for $36 \mathrm{~h}$. The cell lysate was fractionated into cytosolic and mitochondrial portions, and proteins were separated on $15 \%$ SDS-PAGE for cytochrome $c$ immunoblot analysis, and followed by densitometry quantification. The purity of mitochondrial fraction was verified by western blot analysis with anti-VDAC antibody. Data are means \pm SEM of values from 3 independent experiments.

next examined the role of a variety of signal transduction pathways in the modulation of apoptosis. The status of protein kinase B (Akt), c-Jun NH2-terminal kinase (JNK), and p38 mitogen-activated protein kinase (p38 MAPK) was evaluated in A549 cells treated with sulindac and/or simvastatin for 24 and $48 \mathrm{~h}$ (Fig. 4A). The combination of sulindac and simvastatin treatment resulted in a significant time-dependent attenuation of phosphorylated Akt compared to cells treated with either sulindac or simvastatin alone. In contrast, the combined drug treatment resulted in enhanced phosphorylated JNK and p38, compared with single drug treatment. Total protein and GAPDH levels were unaffected by either treatment type.

Combination of sulindac and simvastatin downregulates survivin and induces changes in Bcl-2 families. Members of the IAP and Bcl-2 families are important regulators of the mitochondrial apoptotic pathway. To identify the molecular mechanism underlying apoptosis induced by combined treatment with sulindac and simvastatin, we examined the expression level of the IAP (survivin and XIAP), proapoptotic (Puma and Bim), and anti-apoptotic (Mcl-1 and Bcl-xL) Bcl-2 families, by immunoblot analysis in A549 cells treated with sulindac and/or simvastatin for $36 \mathrm{~h}$. As shown in Fig. 4B, treatment of A549 cells with sulindac and simvastatin resulted in a significant decrease in survivin levels relative to treatment 


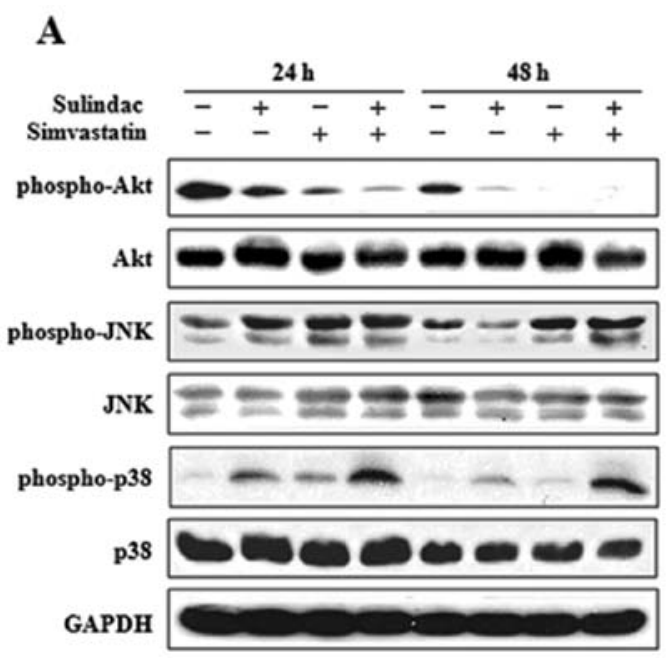

B

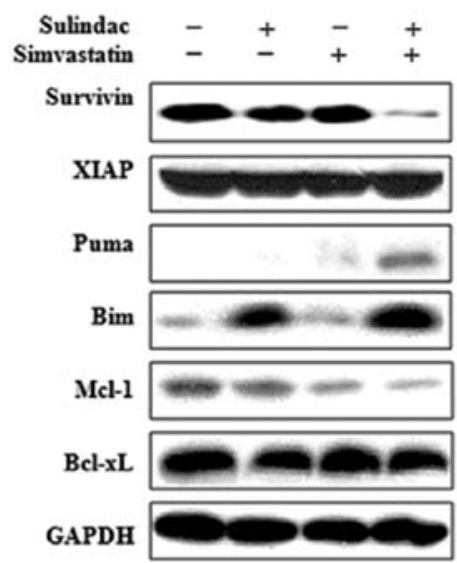

Figure 4. Involvement of different signal transduction pathways by combination treatment with sulindac and simvastatin. (A) Cells were treated with sulindac and simvastatin, alone and in combination, for 24 and $48 \mathrm{~h}$, and the cell lysate was subjected to $12 \%$ SDS-PAGE to measure the expression of phosphorylated Akt, JNK and p38. The same membrane used for anti-phospho antibody staining was stripped and used again with antibodies for Akt, JNK and p38. (B) Cells were treated with sulindac and simvastatin, alone and in combination, for $36 \mathrm{~h}$, and the cell lysate was subjected to $15 \%$ SDS-PAGE to measure the expression of the IAP (survivin and XIAP), proapoptotic (Puma and Bim), and antiapoptic (Mcl-1, Bcl-xL) Bcl-2 families. Equal protein loading was confirmed using GAPDH. Immunoblots are representative of at least 2 independent experiments.

with either drug alone, but it showed no effect on the expression of XIAP. In addition, combination treatment with sulindac and simvastatin increased the expression of the proapoptotic factors Puma and Bim, whereas it resulted in a decrease in the levels of the anti-apoptotic factor Mcl-1. The combination treatment had no effect on the expression level of Bcl-xL.

Elevated ROS contributes to anticancer activity of combination treatment with sulindac and simvastatin. The generation of intracellular ROS is known to occur in lung cancer cells after treatment with sulindac or simvastatin (32). Accordingly, we examined whether the synergistic cytotoxicity of sulindac and simvastatin results from the generation of ROS. After 48-h treatment with $300 \mu \mathrm{M}$ sulindac alone, $5 \mu \mathrm{M}$ simvastatin alone, or a combination of both drugs, cells were loaded with dichlorofluorescein diacetate, and the resulting fluorescence was analyzed on a FACSVantage flow cytometer. We observed a rightward shift of fluorescence signals in cells treated with both sulindac and simvastatin compared with cells treated with either sulindac or simvastatin alone (Fig. 5A). We next tested the effect of the free radical scavenger NAC in sulindac and simvastatin-treated A549 cells. Cells were pretreated with NAC, followed by the addition of sulindac and simvastatin for $24 \mathrm{~h}$. As shown in Fig. 5B, the enhancement of ROS generation by combination treatment with sulindac and simvastatin was abrogated by NAC. Moreover, NAC markedly inhibited combination therapy-induced anticancer activity, as evaluated by the MTT assay (Fig. 5C). Our results indicate that elevated ROS is necessary for the potentiation of cell death in sulindac plus simvastatin-treated cells.

Pretreatment with NAC prevents apoptosis induced by sulindac and simvastatin. To determine whether elevated ROS participates in the apoptosis induced by the combination of sulindac and simvastatin, the proportion of apoptotic cells was determined by Annexin V-propidium iodide staining (Fig. 6A). While the combination of sulindac and simvastatin was associated with Annexin $\mathrm{V}$ positivity in approximately $26.5 \%$ of cells, pretreatment with NAC markedly reduced this rate. Moreover, western blot analysis of A549 cell lysates (Fig. 6B) showed that the combination of sulindac and simvastatin enhanced the expression of the $85-\mathrm{kDa}$ form of PARP, and caspase- $3,-8$ and -9 , whereas pretreatment with NAC blocked this effect. Together, these findings indicate that ROS generation plays a primary role in apoptosis induced by sulindac and simvastatin.

Pretreatment with NAC prevents mitochondrial dysfunction by sulindac and simvastatin. Elevated ROS has been shown to be involved in the mitochondrial apoptotic pathway (33). By flow cytometry analysis performed with the JC-1 fluorescent dye, we investigated the effect of ROS on the mitochondrial transmembrane potential $\left(\Delta \Psi_{\mathrm{m}}\right)$ after combination treatment. As shown in Fig. 7A, we observed JC-1 monomers in 50.2\% of cells treated with the drug combination, whereas the loss of $\Delta \Psi_{\mathrm{m}}$ was significantly reduced in cells pretreated with NAC. Next, we evaluated cytochrome $c$ levels by western blot analysis of mitochondrial and cytosolic fractions (Fig. 7B). Consistent with its effect in offsetting the loss of $\Delta \Psi_{\mathrm{m}}$, pretreatment of A549 cells with NAC abrogated the release of cytochrome $c$ from mitochondria to the cytosol, which is induced by the combination treatment with sulindac and simvastatin. Next, we examined the effect of ROS on the expression of JNK after co-treatment with sulindac and simvastatin (Fig. 7C). Pretreatment with NAC suppressed the increase in the levels of phosphorylated JNK induced by the combination treatment with sulindac and simvastatin. Collectively, these results indicate that pretreatment with NAC suppresses mitochondrial dysfunction induced by sulindac and simvastatin co-treatment.

\section{Discussion}

In the present study, we demonstrated the synergistic effect of a combination of sulindac and simvastatin on apoptosis of A549 lung cancer cells compared to the use of either agent 
A

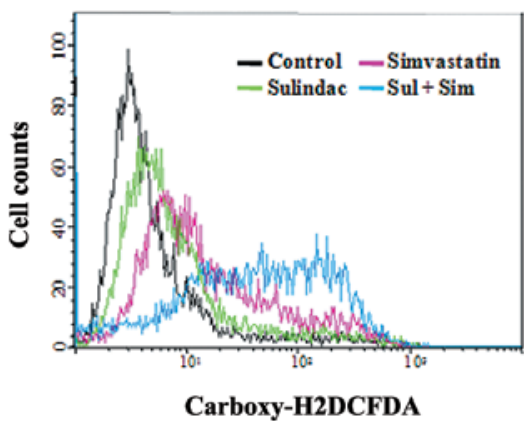

B

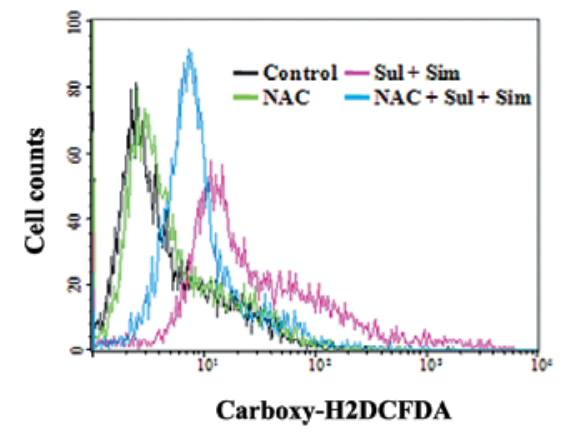

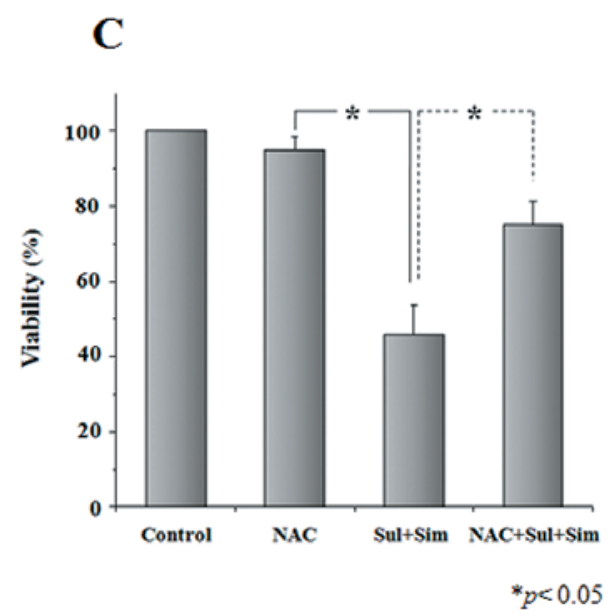

Figure 5. Contribution of ROS generation to the anticancer cell activity of combination therapy in A549 cells. (A) ROS generation. Carboxy- ${ }_{2}$ DCFDA fluorescent signals after incubation with $300 \mu \mathrm{M}$ sulindac alone, $5 \mu \mathrm{M}$ simvastatin alone, or a combination of both drugs for up to $48 \mathrm{~h}$. (B) ROS generation. Cells were treated with sulindac and simvastatin in the presence or absence of $10 \mathrm{mM} \mathrm{NAC} \mathrm{for} 24 \mathrm{~h}$, and then loaded with $10 \mu \mathrm{M}$ carboxy-H $\mathrm{H}_{2} \mathrm{DCFDA}$. Fluorescence measurements in Fig. 5A and B were carried out with a FACSCalibur flow cytometer. Representative data are shown. Numbers indicate the percentage of DCF fluorescence. (C) Cells were treated with sulindac and simvastatin in the presence or absence of NAC for $48 \mathrm{~h}$, and cell viability was determined by MTT assay. The viability of control cells was set at $100 \%$, and cell survival relative to the control is presented. The data represent the mean \pm SD of 3 independent experiments. ${ }^{*}<0.05$ compared to the control.

alone. These findings suggest that a combination of these agents can potentially be used to kill lung cancer cells more effectively and with minimal side-effects, thereby affording a rationale for combining these drugs for the clinical prevention or treatment of lung cancer.

Mitochondria are dynamic organelles that constantly undergo fission and fusion to adapt to the changing conditions of the cell. They play a central role in cellular metabolism and are a major source of ROS generation in cells (34). Recently, the mitochondrial megachannel was suggested to be a source of ROS generation induced by treatment with sulindac and simvastatin (35). Several studies have reported that mitochondrial morphology changes during apoptosis, resulting in the appearance of small, round mitochondrial fragments $(36,37)$. Mitochondria play a major role in many apoptotic responses by coordinating caspase activation through the release of cytochrome $c(38,39)$. Moreover, JNK is known to influence the functions of pro- and anti-apoptotic Bcl-2 family proteins by various mechanisms in the activation of the intrinsic mitochondrial apoptotic pathway (40). Our results demonstrate that the release of cytochrome $c$ into the cytosol activates caspase-9 and JNK signaling, and subsequently leads to the activation of caspase-3. Indeed, cleavage of PARP, a downstream target in this pathway, occurs during sulindac and simvastatin-induced lung cancer cell apoptosis.

Although ROS are essential to cell survival, elevated levels of ROS result in slowed growth, cell cycle arrest, as well as apoptosis or even necrosis (41). Many chemotherapeutic strategies have been designed to significantly increase cellular ROS levels with the goal of inducing irreparable tumor cell damage and death. Intracellular oxidative status has been shown to be important for simvastatin sensitivity, and sulindac is also known to increase ROS levels more efficiently than a selective COX-2 inhibitor (42). Increased ROS induces apoptosis by activating the MAPK and caspase cascades, and/or by disrupting the mitochondrial membrane potential (43). A challenge for novel treatment strategies in lung cancer is the fine-tuning of intracellular ROS signaling for effective therapeutic gain. Accordingly, we investigated the possibility that ROS plays a role in sulindac and simvastatin-induced ROS generation in A549 cells. We demonstrated that, compared to individual treatments, combination treatment with sulindac and simvastatin increased ROS levels, suggesting that the combination of these drugs maintains higher ROS levels. If ROS are indeed involved in apoptosis, ROS quenchers such as antioxidants would be anticipated to prevent apoptosis. Moreover, ROS is a possible initiator of 
A

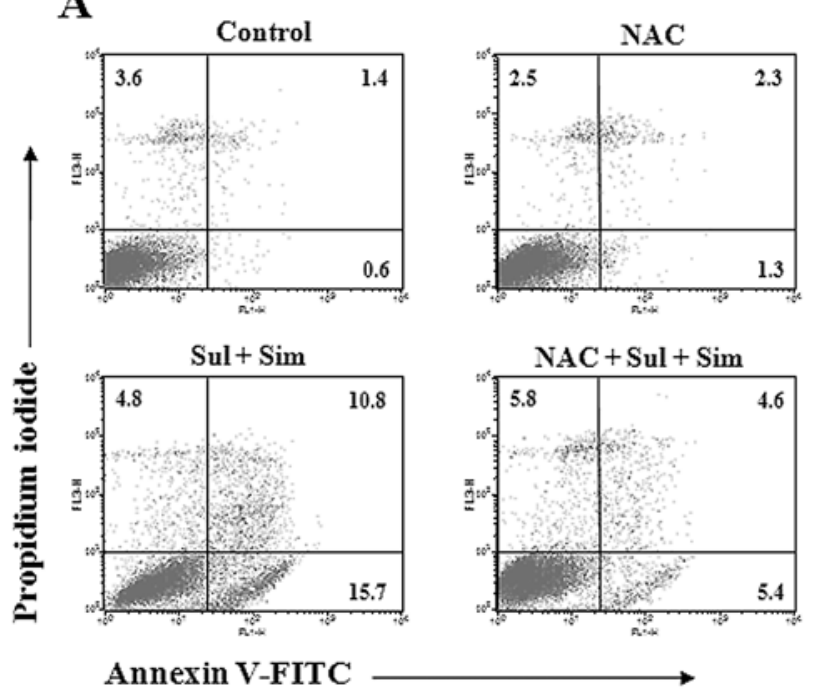

B

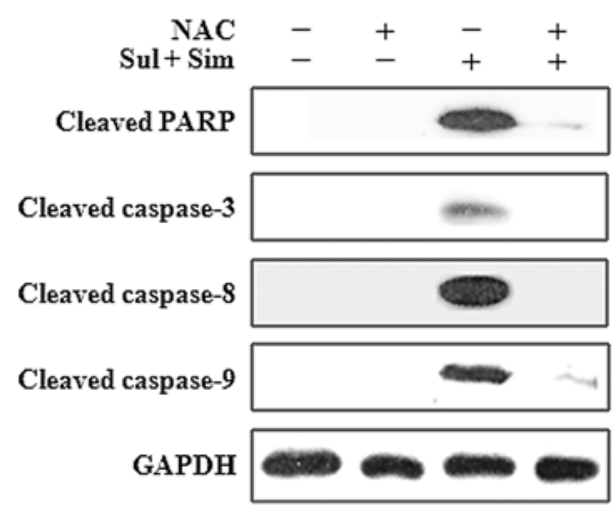

Figure 6. Effect of NAC on sulindac and simvastatin-induced apoptosis. Cells were treated with sulindac and simvastatin in the presence or absence of $10 \mathrm{mM} \mathrm{NAC}$ for $24 \mathrm{~h}$. (A) Apoptosis was evaluated by green fluorescent protein-Annexin $\mathrm{V}+$ propidium iodide. Percentage of Annexin V and propidium iodide positive cells are indicated for each test condition. (B) The cell lysate was subjected to $12 \%$ SDS-PAGE to measure the expression of PARP and caspase- $3,-8$ and -9 . Data are representative of 2 independent experiments.

JNK activation, which is necessary for co-treatment-induced $\Delta \Psi_{\mathrm{m}}$ change. Indeed, we found that sulindac and simvastatininduced apoptosis, mitochondrial dysfunction, and caspase activation were greatly reduced by pretreatment with NAC. These results suggest that, in this model system, ROS generation has a primary role in the induction of apoptosis by sulindac and simvastatin.

We found that activation of caspases, mitochondrial cytochrome $c$ release, and change in $\Delta \Psi_{\mathrm{m}}$ occurred during sulindac and simvastatin-induced apoptosis. JNK and p38 MAPK were activated in combination with sulindac and simvastatin, but only JNK activation was necessary for the co-treatment-induced change in $\Delta \Psi_{\mathrm{m}}$ and lung cancer cell death. Pretreament with NAC reduced co-treatment-induced activation of caspases and lung cancer cell death. On the basis of our findings, we suggest that ROS and JNK are involved in sulindac and simvastatininduced lung cancer cell apoptosis. Our findings afford insight
A

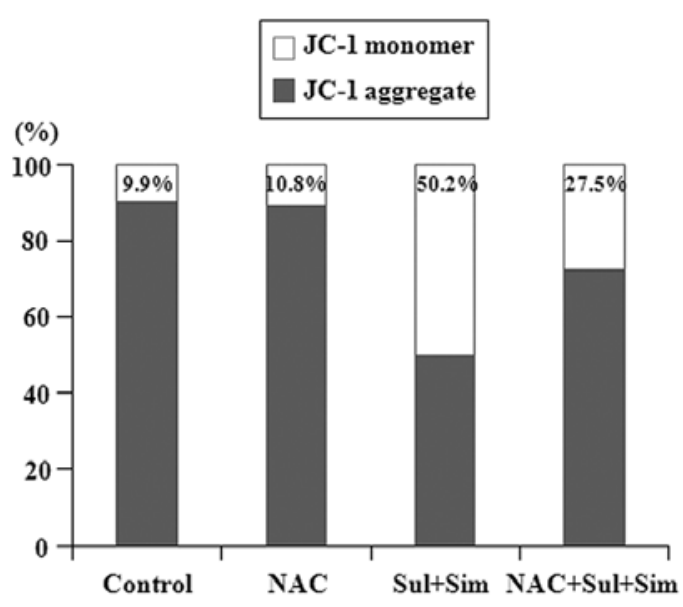

B

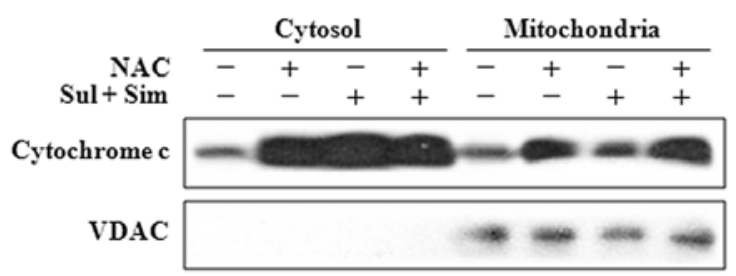

C

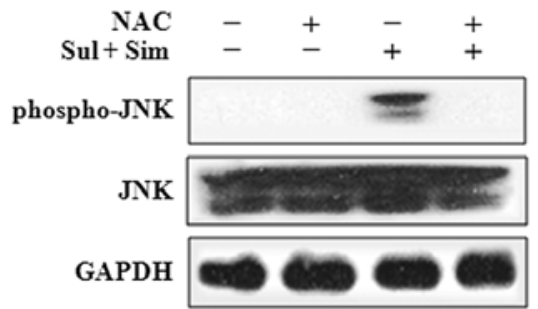

Figure 7. Effect of NAC on mitochondrial dysfunction and JNK in combination-treated cells. Cells were treated with sulindac and simvastatin in the presence or absence of $10 \mathrm{mM}$ NAC for $36 \mathrm{~h}$. (A) Cells were stained with $10 \mu \mathrm{g} / \mathrm{ml}$ of JC-1 and analyzed by flow cytometry. Percentage of JC-1 monomer are indicated for each test condition. Each panel is representative of 3 identical experiments. (B) Cell lysate was fractionated into cytosolic and mitochondrial portions, and proteins were separated on 15\% SDS-PAGE for cytochrome $c$ immunoblot analysis, and followed by densitometry quantification. The purity of mitochondrial fraction was verified by western blot analysis with anti-VDAC antibody. Data are means \pm SEM of values from 3 independent experiments. (C) The cell lysate was subjected to $12 \%$ SDS-PAGE to measure the expression of JNK. Data are representative of 2 individual experiments.

into ROS-mediated signaling in tumor cells, as well as provide a basis for the design of novel improved strategies for the treatment of lung cancer.

In conclusion, our results indicate that combination treatment with sulindac and simvastatin augments their apoptotic potential in lung cancer cells through ROS-dependent mitochondrial dysfunction. Taken together, these results indicate that sulindac and simvastatin are clinically promising therapies for the treatment of lung cancer. Our data elucidate the possible mechanism of action for sulindac and simvastatin in effecting lung cancer cell death, and postulate their co-treatment as a chemopreventive approach in lung cancer. 


\section{Acknowledgements}

This study was supported by a grant of the Korean Health Technology R\&D Project, Ministry of Health and Welfare (A120152), Republic of Korea.

\section{References}

1. Tanaka K, Iwamoto S, Gon G, Nohara T, Iwamoto M and Tanigawa N: Expression of survivin and its relationship to loss of apoptosis in breast carcinomas. Clin Cancer Res 6: 127-134, 2000.

2. Velmurugan B, Mani A and Nagini S: Combination of S-allylcysteine and lycopene induces apoptosis by modulating $\mathrm{Bcl}-2, \mathrm{Bax}, \mathrm{Bim}$ and caspases during experimental gastric carcinosenesis. Eur J Cancer Prev 14: 387-393, 2005.

3. Lin J, Hsiao PW, Chiu TH and Chao JI: Combination of cyclooxygenase- 2 inhibitors and oxaliplatin increases the growth inhibition and death in human colon cancer cells. Biochem Pharmacol 70: 658-667, 2005.

4. Banerjee S, Zhang Y, Ali S, Bhuiyan M, Wang Z, Chiao PJ, Philip PA, Abbruzzese J and Sarkar FH: Molecular evidence for increased antitumor activity of gemcitabine by genistein in vitro and in vivo using an orthotopic model of pancreatic cancer. Cancer Res 65: 9064-9072, 2005.

5. Khor TO, Keum YS, Lin W, Kim JH, Hu R, Shen G, Xu C, Gopalakrishnan A, Reddy B, Zheng X, Conney AH and Kong AN: Combined inhibitory effects of curcumin and phenethyl isothiocyanate on the growth of human PC-3 prostate xenografts in immunodeficient mice. Cancer Res 66: 613-621, 2006.

6. Giardiello FM, Spannhake EW, DuBois RN, Hylind LM, Robinson CR, Hubbard WC, Hamilton SR and Yang VW: Prostaglandin levels in human colorectal mucosa: effects sulindac in patients with familial adenomatous polyposis. Dig Dis Sci 43: 311-316, 1998.

7. Shiff SJ, Qiao L, Tsai LL and Rigas B: Sulindac sulfide, an aspirin-like compound, inhibits proliferation, causes cell cycle quiescence, and induces apoptosis in HT-29 colon adenocarcinoma cells. J Clin Invest 96: 491-503, 1995.

8. Chan TA, Morin PJ, Vogelstein B and Kinzler KW: Mechanism underlying nonsteroidal antiinflammatory drug-mediated apoptosis. Proc Natl Acad Sci USA 95: 681-686, 1998.

9. Williams CS, Mann M and DuBios RN: The role of cyclooxygenases in inflammation, cancer, and development. Oncogene 18: 7908-7916, 1999.

10. Thompson HJ, Jiang C, Lu J, Mehta RG, Piazza GA, Paranka NS, Pamukcu R and Ahnen DJ: Sulfone metabolite of sulindac inhibits mammary carcinogenesis. Cancer Res 57: 267-271, 1997.

11. Piazza GA, Rahm AL, Krutzsch M, Sperl G, Paranka NS, Gross PH, Brendel K, Burt RW, Alberts DS and Pamukcu R Antineoplastic drugs sulindac sulfide and sulfone inhibit cell growth by inducing apoptosis. Cancer Res 55: 3110-3116, 1995.

12. Tegeder I, Pfeilschifter J and Geisslinger G: Cyclooxygenaseindependent actions of cyclooxygenase inhibitors. FASEB J 15 2057-2072, 2001.

13. Seo SK, Lee HC, Woo SH, Jin HO, Yoo DH, Lee SJ, An S, Choe TB, Park MJ, Hong SI, Park IC and Rhee CH: Sulindacderived reactive oxygen species induce apoptosis of human multiple myeloma cells via p38 mitogen activated protein kinase-induced mitochondrial dysfunction. Apoptosis 12: 195-209, 2007.

14. Goldstein JL and Brown MS: Regulation of the mevalonate pathway. Nature 343: 425-430, 1990.

15. Chan KK, Oza AM and Siu LL: The statins as anticancer agents. Clin Cancer Res 9: 10-19, 2003.

16. Demierre MF, Higgins PD, Gruber SB, Hawk E and Lippman SM: Statins and cancer prevention. Nat Rev Cancer 5: 930-942, 2005.

17. Mantha AJ, Hanson JE, Goss G, Lagarde AE, Lorimer IA and Dimitroulakos J: Targeting the mevalonate pathway inhibits the function of the epidermal growth factor receptor. Clin Cancer Res 11: 2398-2407, 2005.

18. Wong WW, Dimitroulakos J, Minden MD and Penn LZ: HMB-CoA reductase inhibitors and the malignant cell: the statin family of drugs as triggers of tumor-specific apoptosis. Leukemia 16: 508-519, 2002.
19. Holstein SA and Hohl RJ: Synergistic interaction of lovastatin and paclitaxel in human cancer cells. Mol Cancer Ther 1: 141-149, 2001.

20. Feleszko W, Mlynarczuk I, Olszewska D, Jalili A, Grzela T, Lasek W, Hoser G, Korczak-Kowalska G and Jakobisiak M: Lovastatin potentiates antitumor activity of doxorubicin in murine melanoma via an apoptosis-dependent mechanism. Int J Cancer 100: 111-118, 2002.

21. Khanzada UK, Pardo OE, Meier C, Downward J, Seckl MJ and Arcaro A: Potent inhibition of small-cell lung cancer cell growth by simvastatin reveals selective functions of Ras isoforms in growth factor signaling. Oncogene 25: 877-887, 2006.

22. Kozar K, Kaminski R, Legat M, Kopec M, Nowis D, Skierski JS, Koronkiewicz M, Jakobisiak M and Golab J: Cerivastatin demonstrates enhanced antitumor activity against human breast cancer cell lines when used in combination with doxorubicin or cisplatin. Int J Oncol 24: 1149-1157, 2004.

23. Wallace JL: Nonsteroidal anti-inflammatory drugs and gastroenteropathy: the second hundred years. Gastroenterology 112: 1000-1016, 1997.

24. Solomon SD, McMurray JJ, Pfeffer MA, Wittes J, Fowler R, Finn R, Anderson WF, Zauber A, Hawk E and Bertagnolli M: Cardiovascular risk associated with celecoxib in a clinical trial for colorectal adenoma prevention. N Eng J Med 352: 1071-1080, 2005

25. Psaty BM and Potter JD: Risks and benefits of celecoxib to prevent recurrent adenomas. N Engl J Med 355: 950-952, 2006.

26. Jalving M, Koorntra JJ, De Jong S, De Vries EG and Kleibeuker JH: Review article: the potential of combinational regimen with non-steroidal anti-inflammatory drugs in the chemoprevention of colorectal cancer. Aliment Pharmacol Ther 21: 321-339, 2005.

27. Xiao $\mathrm{H}$ and Yang CS: Combination regimen with statins and NSAIDs: a promising strategy for cancer chemoprevention. Int J Cancer 123: 983-990, 2008.

28. Suh N, Reddy BS, DeCastro A, Paul S, Lee HJ, Smolarek AK, So JY, Simi B, Wang CX, Janakiram NB, Steele V and Rao CV: Combination of atorvastatin with sulindac or naproxen profoundly inhibits colonic adenocarcinomas by suppressing the p65/ $\beta$-catenin/cyclin D1 signaling pathway in rats. Cancer Prev Res (Phila) 4: 1895-1902, 2011.

29. Lowry OH, Rosebrough NJ, Farr AL and Randall RJ: Protein measurement with the Folin phenol reagent. J Biol Chem 193: 265-275, 1951.

30. Wolf CM and Eastman A: The temporal relationship between protein phosphatase, mitochondrial cytochrome c release. Exp Cell Res 247: 505-513, 1999.

31. Smiley ST, Reers M, Mottola-Hartshorn C, Lin M, Chen A, Smith TW, Steele GD Jr and Chen LB: Intracellular heterogeneity in mitochondrial membrane potentials revealed by a J-aggregate-forming lipophilic cation JC-1. Proc Natl Acad Sci USA 88: 3671-3675, 1991.

32. Park JH, Kim EJ, Jang HY, Shim H, Lee KK, Jo HJ, Kim HJ, Yang SH, Jeong ET and Kim HR: Combination treatment with arsenic trioxide and sulindac enhances apoptotic cell death in lung cancer cells via activation of oxidative stress and mitogenactivated protein kinases. Oncol Rep 20: 379-384, 2008.

33. Ralph SJ, Rondriguez-Enriquez S, Neuzil J, Saavedra E and Moreno-Sanchez R: The causes of cancer revisited: 'mitochondrial malignancy' and ROS-induced oncogenic transformation - why mitochondria are targets for cancer therapy. Mol Aspects Med 31: 145-170, 2010.

34. Copeland WC, Wachsman JT, Johnson FM and Penta JS: Mitochondrial DNA alterations in cancer. Cancer Invest 20: 557-569, 2002.

35. Seo SK, Lee HC, Woo SH, Jin HO, Yoo DH, Lee SJ, An S, Choe TB, Park MJ, Hong SI, Park IC and Rhee CH: Sulindac-derived reactive oxygen species induce apoptosis of human multiple myeloma cells via p38 mitogen activated protein kinase-induced mitochondrial dysfunction. Apoptosis 12: 195-209, 2007.

36. Frank S, Gaume B, Bergmann-Leitner ES, Leitner WW, Robert EG, Catez F, Smith CL and Youle RJ: The role of dynamic-related protein 1, a mediator of mitochondrial fission, in apoptosis. Dev Cell 1: 515-525, 2001.

37. Karbowski M, Lee YJ, Gaume B, Jeong SY, Frank S, Nechushtan A, Santel A, Fuller M, Smith CL and Youle RJ: Spatial and temporal association of Bax with mitochondrial fission sites, Drp1, and Mfn2 during apoptosis. J Cell Biol 159: 931-938, 2002. 
38. Green DR and Reed JC: Mitochondria and apoptosis. Science 281: 1309-1312, 1998.

39. Li LY, Luo $X$ and Wang X: Endonuclease G is an apoptotic DNase when released from mitochondria. Nature 412: 95-99, 2001.

40. Tournier C, Hess P, Yang DD, Xu J, Turner TK, Nimnual A, Bar-Sagi D, Jones SN, Flavell RA and Davis RJ: Requrement of JNK for stress-induced activation of the cytochrome c-mediated death pathway. Science 288: 870-874, 2000.
41. Burdon RH: Control of cell proliferation by reactive oxygen species. Biochem Soc Trans 24: 1028-1032, 1996.

42. Minami T, Adachi M, Kawamura R, Zhang Y, Shinomura Y and Imai K: Sulindac enhances the proteasome inhibitor bortezomib-mediated oxidative stress and anticancer activity. Clin Cancer Res 11: 5248-5256, 2005.

43. Fiers W, Beyaert R, Declercq W and Van den Abeele P: More than one way to die: apoptosis, necrosis and reactive oxygen damage. Oncogene 18: 7719-7730, 1999. 